\title{
TWICTHPLAYSPOKEMON - MODDING COMO EXPERIMENTO DA CULTURA PARTICIPATIVA
}

\author{
TwicthPlaysPokemon - modding as an experiment of participatory culture \\ TwicthPlaysPokemon - modding como experimento de la cultura participativa
}

\author{
Dario Mesquita \\ Universidade Federal de São Carlos (UFSCar) \\ dario@ufscar.br
}

Sérgio Nesteriuk

Universidade Anhembi Morumbi (UAM)

nesteriuk@hotmail.com

\section{Resumo}

Este artigo discute o canal TwicthPlaysPokemon, do serviço de vídeo sob demanda Twicth.TV, em que jogos da franquia Pokémon são modificados para funcionar sob uma plataforma de vídeo hackeada por usuários, buscando compreender uma dimensão intensificada da criação colaborativa por parte dos fãs. Para tanto, é feito um estudo sobre o conceito de cultura participativa e convergência de mídia, para posteriormente abordar a prática de hack e modding no contexto do objeto de estudo.

Palavras-chave: cultura participativa; convergência de mídia; videogame; modding; hack.

\section{Abstract}

This paper discusses the TwicthPlaysPokemon, a channel on the VOD service Twicth.TV, in which games of the Pokémon franchise are modified to operate under a user hacked video platform. It tries to understand an intensified dimension of fan creative collaboration. For this purpose, there is a study on the concept of participatory culture and media convergence, to later approach the practice of hacking and modding in the context of the object of study.

Key words: participatory culture; media convergence; videogame; modding; hack.

\section{Resumen}

Este artículo trata sobre TwicthPlaysPokemon, un canal del servicio VOD Twicth.TV, en el que los juegos de la franquicia Pokémon se modifican para operar bajo una plataforma de video pirateada por el usuario. Intenta comprender una dimensión intensificada de la colaboración creativa de los fanáticos. Para este propósito, hay un estudio sobre el concepto de cultura participativa y convergencia de medios, para luego abordar la práctica de piratería y modificación en el contexto del objeto de estudio.

Palabras clave: cultura participativa; convergencia de medios; video juego; modding; hack. 


\section{INTRODUÇÃO}

Criada em 2011, a plataforma de vídeo sob demanda Twitch.TV surgiu como um spin-off da seção de jogos de outra plataforma de vídeo sob demanda chamada Justin.TV, lançada em 2007 e encerrada em 2014 ao ser comprada pela Amazon. O que antes era apenas uma seção especializada na transmissão ao vivo de partidas de jogadores amadores e profissionais em campeonatos mundiais de e-Sports, tornou-se maior que a Justin.TV, ganhando sua independência enquanto plataforma própria. Atualmente, a Twitch.TV é o maior serviço de streaming de jogos, com mais de 100 milhões de usuários cadastrados, e cerca de 4 milhões de transmissões diárias ${ }^{1}$.

Segundo Hamilton (et al. 2014), a plataforma Twitch.TV é mais que um serviço audiovisual na internet, é um espaço de socialização que combina ferramentas de streaming de vídeo ao vivo com salas de bate papo para criar uma comunidade em torno da prática de compartilhar suas experiências com videogames. Ela é composta principalmente por usuários que buscam acesso a "[...] um conteúdo único de uma transmissão em particular, e que gostam de interagir e participar de uma comunidade voltada para o streaming"2 (HAMILTON et al., 2014, p. 1315, tradução nossa). Conforme observado, a plataforma pode ser considerada uma comunidade participativa, que se articula como uma rede de usuários caracterizada pela participação aberta (sem grande interferência da empresa mantenedora) e pelo encorajamento em compartilhar suas atividades. Desta forma, procura-se criar um senso de pertencimento à comunidade por meio da troca de experiências em torno da prática de jogar videogames.

Hamilton (et al. 2014) destaca a organização emergente das relações na Twitch.TV, em que usuários exercem papéis distintos em diferentes momentos para o fortalecimento destas comunidades, tais como no engajamento com outros usuários, a fim de incentivar a participação pela postagem de vídeos, e na moderação das salas de bate-papo das transmissões.

Nesse contexto, foi criado de forma anônima em 2014 o canal TwicthPlaysPokemon ${ }^{3}$ (Fig. 1), que surgiu como um experimento participativo que explorou os limites técnicos da plataforma e criou uma experiência colaborativa até então inédita na Twitch.TV. Além de

\footnotetext{
${ }^{1}$ Disponível em: https://twitchtracker.com/statistics. Acesso em: 22 ago. 2018

2 No original: [...] unique content of a particular stream, and they like being interacted with and participating in that stream's community.

${ }^{3}$ Disponível em: https://go.twitch.tv/twitchplayspokemon. Acesso em: 06 out. 2017.
} 
transmitir partidas de jogos da franquia ${ }^{4}$ Pokémon, o canal permite aos usuários jogá-los de forma coletiva por meio de comandos de texto específicos digitados no bate-papo. O primeiro jogo transmitido foi Pokémon $\operatorname{Red}^{5}$, lançado originalmente em 1996 para o console portátil GameBoy, da Nintendo. A partida durou cerca de 190 horas, e chegou a contar com mais de 100 mil usuários simultâneos, o que sobrecarregou o servidor da plataforma na época (GUSMÃO, 2014). Desde então, o canal vem realizando transmissões regulares com outros jogos da franquia.

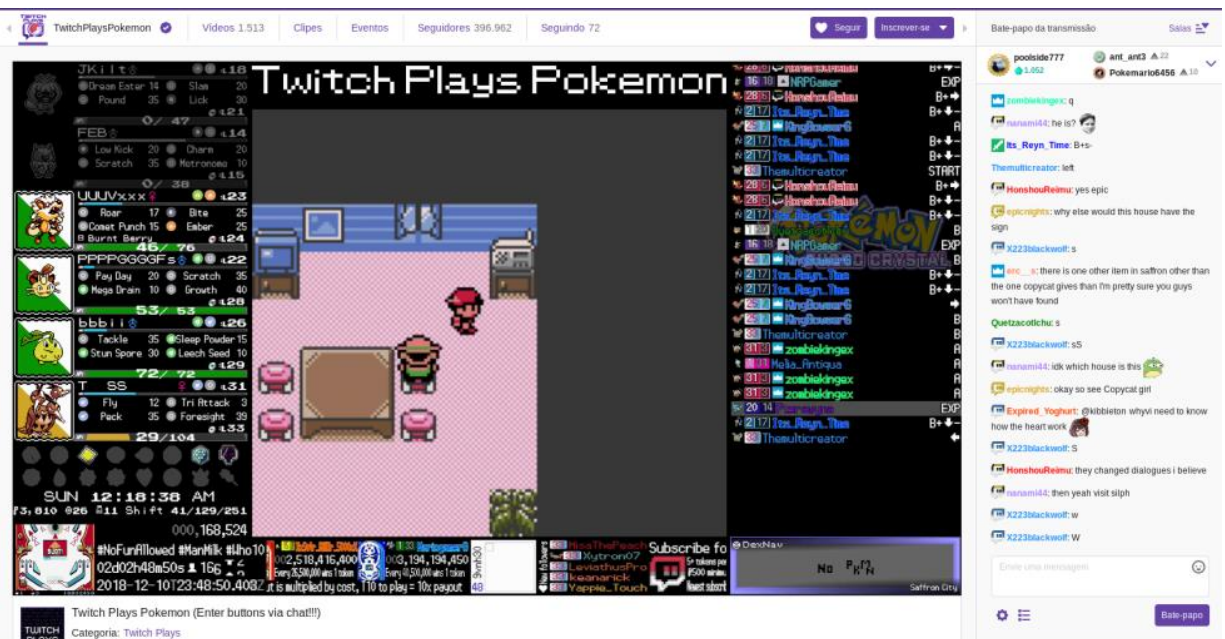

Figura 1 - Screenshot do canal TwicthPlaysPokemon, executando o jogo Pokémon Crystal (Nintendo, 2000) Fonte: Reprodução do autor. Data de captura da tela: 10 dez. 2018

A interface do canal (Fig. 1), retrabalha a interface original do jogo, transmitido no centro da tela, onde são sobrepostos avisos e estatísticas sobre a partida. No canto direto, encontra-se a sala de bate-papo, em que os comandos são digitados meio ao diálogo entre os usuários.

Além de ser uma modificação sobre os jogos da franquia Pokémon, TwicthPlaysPokemon também funciona como uma forma de hacking sobre as funcionalidades da própria plataforma de vídeo Twicth.TV. Segundo Sotamma (2003), interferências como essas não são apenas um reflexo da acessibilidade de ferramentas para tal prática, englobando dinâmicas que são consequência do que Jenkins (2003) denomina de cultura participativa na qual os limites entre realizadores e público ficam tênues no ambiente midiático. Entretanto, enquanto a colaboração na cultura participativa cria produtos bem delimitados, como contos

\footnotetext{
${ }^{4}$ Franquia é compreendida no presente artigo como uma "estrutura corporativa para a produção de mídia que tem uma longa história" (JENKINS, 2011, s/p), atrelada a uma gerência de marca sobre a propriedade intelectual por um longo período de tempo, utilizando-se de estratégias comerciais para mover símbolos para canais de mídia e produtos derivados, com a finalidade de expandir o escopo e diversificar o público-alvo.

${ }^{5}$ Em Pokémon Red, o jogador assume o papel de um aspirante a treinador de criaturas conhecidas como Pokémons, com o objetivo de capturá-los, treiná-los e batalhar com eles.
} 
escritos, filmes curtos ou ações mais livres como discussões em fóruns, TwicthPlaysPokemon é caracterizado por uma complexidade emergente com o objetivo de concluir uma partida de jogo. Adjacente a essa dimensão do canal, outras dinâmicas são estabelecidas e negociadas na manutenção da experiência.

Desse modo, o presente artigo discute a prática do hacking da plataforma Twicth.TV, a fim de modificar as funcionalidades originais de jogos da franquia Pokémon, pela ótica da cultura participativa. Por essa perspectiva, busca-se melhor compreender as diferentes dinâmicas que contextualizam a criação do TwicthPlaysPokemon, principalmente seu caráter emergente enquanto experiência colaborativa.

Para tanto, primeiro se fará um estudo sobre o conceito de cultura participativa, para posteriormente abordar a prática do modding em videogames, uma expressão derivada do verbo inglês modify que se refere às modificações feitas por usuários na estética ou no código fonte de jogos digitais, para troca de texturas e objetos, ou modificar a funcionalidade de suas regras (SOTAMMA, 2003). Em seguida, é feita uma breve análise sobre o canal TwicthPlaysPokemon enfatizando determinadas práticas dos usuários. Ao final, serão feitas considerações sobre o objeto de estudo e seus conceitos atinentes, apontando-se novos caminhos de pesquisa, sobretudo no que tange experimentos emergentes massivos no contexto da cultura participativa.

\section{CULTURA PARTICIPATIVA E VIDEOGAMES}

Como comenta Jenkins (2003), não é tão recente o entendimento de que o público seja ativo no consumo de mídias. Desde a década de 1970, pesquisas influenciadas pelos Estudos Culturais ingleses, por exemplo, enfatizam como o público se apropria dos conteúdos que circulam nos meios de comunicação de diferentes formas. Assim, foram superadas as interpretações do consumidor de mídia como elemento passivo mediante os estímulos midiáticos, pois, os sujeitos possuiriam capacidade crítica para reinterpretar as mensagens veiculadas, ou mesmo teriam meios alternativos para se manifestar nas mídias.

Atualmente, a produção de conteúdo por parte do público se intensificou por meio da internet e de suas plataformas de compartilhamento. Produtores e consumidores que em outra época ocupavam papéis separados agora interagem e dialogam mutuamente em dinâmicas que sofrem constantes transformações (JENKINS, 2009). São práticas participativas que se 
intensificaram em consequência de alguns fatores, como apontados por Jenkins (2003, s/p, tradução nossa):

(1) novas ferramentas e tecnologias permitem aos consumidores arquivar, anotar, apropriar e reciclar conteúdo de mídia;

(2) uma variedade de subculturas que promovem a produção de mídia Do-ItYourself (DIY), um discurso que molda como os consumidores implantaram essas tecnologias;

(3) as tendências econômicas que favorecem os conglomerados de mídia horizontalmente integrados incentivarem o fluxo de imagens, ideias e narrativas em vários canais de mídia e exigirem espectadores mais ativos. ${ }^{6}$

O autor também destaca a forma como múltiplos suportes midiáticos convergem em dimensões distintas: pelo fluxo de conteúdos que trafega entre os meios; pela cooperação entre diferentes mercados de mídia em torno de uma propriedade intelectual; e pelo movimento migratório do público entre diferentes telas na busca de novas experiências de entretenimento e formas de se expressar em um ambiente de comunicação em rede.

Desse modo, a própria noção de mídia é modificada, em que a relação entre a materialidade do meio e a linguagem perde importância em relação ao conteúdo em si e suas possibilidades de integração entre diferentes mídias, a fim de viabilizar a participação do público. Os meios de comunicação deixam de ser encarados como aparatos isolados para serem pensados e articulados como plataformas modulares, de intermediação não apenas de mensagens, mas também de práticas e serviços, integradas em uma rede de produção de conteúdos focados na experiência do usuário. Como afirma Jenkins (2009, p. 28), a convergência de mídia, como podemos denominar esse fenômeno, “[...] representa uma transformação cultural, à medida que consumidores são incentivados a procurar novas informações e fazer conexões em meio a conteúdos midiáticos dispersos".

Como exemplo desse cenário, podemos destacar os agrupamentos de fãs, que atuam "na produção e na distribuição de mídia para atender a seus interesses coletivos" (JENKINS et at., 2014, p. 24), sobretudo no que tange propriedades intelectuais do entretenimento de mídia. Nesse âmbito, são produzidas obras ficcionais de fãs em texto (fanfic) e em vídeo (fan film),

\footnotetext{
${ }^{6}$ No original: (1) new tools and technologies enable consumers to archive, annotate, appropriate, and recirculate media content

(2) a range of subcultures promote Do-It-Yourself (DIY) media production, a discourse that shapes how consumers have deployed those technologies

(3) economic trends favoring the horizontally integrated media conglomerates encourage the flow of images, ideas, and narratives across multiple media channels and demand more active modes of spectatorship.
} 
por apropriações de imagens por meio de memes $^{7}$, metatextos em wikis ${ }^{8}$ que catalogam o histórico de mundos ficcionais, ou por meio de jogos modificados ou criados pelo público, com ou sem consentimentos dos detentores dos diretos autorais.

Tais práticas são encontradas no universo da franquia Pokémon. Criada pelo game designer Satoshi Tajiri, a franquia nasce em 1989 com o lançamento do primeiro videogame homônimo para o console portátil Game Boy, em uma parceria entre a Nintendo e a empresa desenvolvedora Game Freak. A partir da premissa que gira em torno da caça e do treinamento de monstros chamados Pokémons (utilizados em batalhas competitivas), já foram desenvolvidas narrativas seriadas para quadrinhos e televisão (com mais de 900 episódios), mais de vinte filmes para cinema e home video, além de cerca de dezessete jogos para consoles e um jogo de cartas que possui competições profissionais promovidas em diferentes países (NEDELCHEVA, 2016).

São mais de 800 pokémons existentes no universo da franquia, cada qual com suas estratégias de combate extensamente discutidas pelos fãs, que igualmente organizam eventos como "[..] torneios, concursos e exposições, gerenciam informações em bancos de dados, e ainda mais - criam artes, incluindo histórias em quadrinhos, brinquedos de pelúcia, e até novos videogames!"9 (NEDELCHEVA, 2016, p. 2, tradução nossa). Dentre as criações dos usuários, podemos destacar o PokeMMO ${ }^{10}$ (Fig. 2), um jogo massivo online gratuito para computadores. Lançado em 2012, o fangame apresenta sons e gráficos que remetem aos primeiros títulos da franquia ${ }^{11}$, e se propõe a criar uma experiência de jogo em um vasto mundo online, no qual os usuários caçam pokémons e batalham em tempo real com outros jogadores.

\footnotetext{
${ }^{7}$ Utiliza-se a palavra meme para designar uma imagem, frase ou vídeo que utiliza códigos comunicacionais que se propagam potencialmente nas redes, ou seja, "que são replicados de um usuário das redes para outros, que por sua vez também o replicam até que o conteúdo atinja um grande número de pessoas. [...] Em geral, os memes trabalham com comicidade e ironia" (CERETTA, 2016, p. 3).

8 Plataforma online para criação de websites colaborativos enciclopédicos de temáticas segmentadas, onde qualquer usuário registrado por criar, editar e moderar os conteúdos publicados.

${ }^{9}$ No original: [...] tournaments, contests and exhibitions, arrange information in databases, and all the more - create artwork, including comic books, plush toys, even new video games!

${ }^{10}$ Mais informações: https://pokemmo.eu/

${ }^{11}$ Conferir: https://youtu.be/2UJnjYXu5x4. Acesso em: 07 set. 2018.
} 


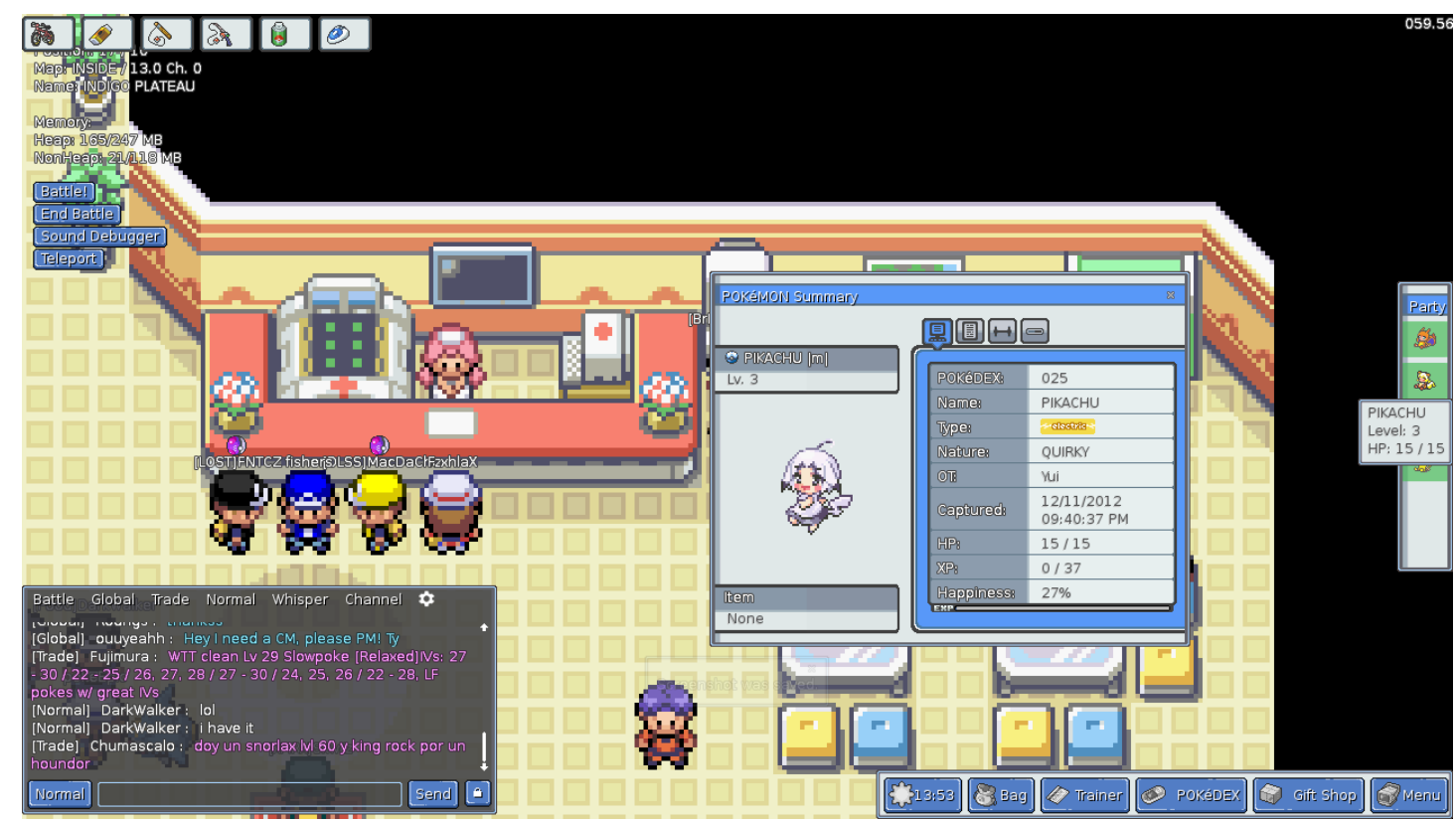

Figura 2 - Screenshot do jogo PokeMMO, criado por fãs da franquia Pokémon Fonte: http://i.imgur.com/OGKFO.png. Acesso em: $10 \mathrm{dez} .2018$.

Apesar de haver uma extensa produção do público em torno da franquia sem interferência das empresas detentoras dos direitos autorias, a relação com a Nintendo pode ser de conflito em determinadas situações. Em 2016, por exemplo, a empresa notificou os desenvolvedores do fangame Pokémon Uranium para remover o link para download uma semana após o seu lançamento (GOOD, 2016), quando o jogo já havia sido baixado mais de 1.5 milhões de vezes. O jogo demandou mais de nove anos de produção, e ganhou grande destaque junto à comunidade de jogadores da franquia. Mesmo após a notificação e a retirada do link do site de origem, o fangame continua a ser compartilhado por outros canais entre os jogadores (GOOD, 2016).

Como aborda Jenkins (et al., 2016), atritos dessa natureza são relativamente comuns no ambiente dos videogames, especialmente nos casos de práticas de modding - condenadas por algumas empresas por criarem projetos "que trilham desconfortavelmente perto de seus próprios planos de produção ou seguem em direções não aprovadas pelos detentores de direito" (idem, p. 89). Por outro lado, há empresas que incentivam o modding, disponibilizando o código ou ferramentas já direcionadas para modificações. Estas compreendem as vantagens econômicas por meio da geração e do compartilhamento de 
conteúdo de forma gratuita por parte do público. Ou seja, as apropriações podem ser estratégicas para o fortalecimento do relacionamento da marca com o público e na geração de novos produtos, mantendo a marca ou franquia por mais tempo em evidência.

Um exemplo dessa estratégia é Counter-Strike (CS), um jogo de tiro em primeira pessoa multi-player online que surgiu em 1999 como uma modificação do título Half-Life (1998) da desenvolvedora Valve Software, que acabou por adquirir o jogo modificado em 2000 e criar três continuações (a última, foi lançada em 2012, denominada CS: Global Offensive). Hoje, Counter-Strike é um dos principais jogos do gênero, ganhando mais destaque do que o próprio jogo originalmente desenvolvido pela Valve Sotware. Porém, é importante observar que uma das primeiras empresas a incentivar essa prática foi a id Software, que em 1997 liberou o código-fonte de seu jogo Doom (1993), legitimando uma prática dos fãs que vinha ocorrendo desde seu lançamento (SCACCHI, 2010) ${ }^{12}$.

Como observa Gregolin (2010, p. 52), o modding remete à cultura hacker da década de 1980, quando programadores e seus grupos exibiam "suas habilidades em modificar o código do jogo, gerando pequenas assinaturas nos programas - conhecidos como 'demos"”, que eram compartilhadas discretamente entre jogadores em mídia física e em redes online disponíveis na época. Champion (2013) comenta que o modding passou a ocupar um papel de protagonismo na indústria de jogos, pois, além de inspirar usuários a se tornarem futuros profissionais, também prolonga a vida útil de muitos jogos, e suas franquias, pela criação de conteúdo adicional gratuito para os jogadores.

Nesse sentido, TwicthPlaysPokemon surge dessa convergência do modding com a cultura participativa dos fãs em rede. Não se trata exatamente de um fangame, por não ser um jogo novo criado por fãs, mas de uma modificação que explora novas formas de apropriação pelo público. Uma experiência que também pode ser encarada como hacking de mídia, no qual uma plataforma de vídeo sob demanda é transformada para o controle coletivo das ações de um jogo, que foi projetado originalmente para ser jogado apenas por um ou dois jogadores simultaneamente.

\section{TWICTHPLAYSPOKEMON - CONVERGÊNCIAS E MODDING}

\footnotetext{
${ }^{12}$ Doom foi um dos primeiros jogos de tiro em primeira pessoa, conhecido por sua violência gráfica e temática satanista. E existe uma grande variedade de mods do jogo original, envolvendo temáticas de pirata ou crossover com os monstros da franquia cinematográfica Aliens.
} 
Antes de abordar a prática do modding, Sotamma (2003) fala da importância de o distingui-lo do sentido que o hacking tomou no campo dos videogames atualmente. $\mathrm{O}$ autor compreende o hacking, de maneira geral, como o ato de alterar e manipular o projeto de um artefato e sua finalidade pelo envolvimento nesta ação. Machado (2010) complementa essa compreensão ao enfatizar que a cultura hacker tem como cerne os princípios que as informações devem ser livres e a computação utilizada como um meio para se manter o direito de livre expressão e à privacidade dos sujeitos na rede, partindo do entendimento que o hacking é um trabalho conjugado ao lazer, sem fins lucrativos, e com foco em ações colaborativas.

Porém, como aponta Sotamma (2003), o hacking no contexto dos jogos acabou obtendo outro sentido, tornando-se sinônimo de cheat (trapaça), ou seja, um ato de manipulação do design do jogo a fim de burlar as regras em benefício próprio. Em partidas online, por exemplo, é comum ofender e denunciar jogadores com ótimo desempenho por serem possíveis hackers, utilizando comandos pré-programados para burlar os desafios.

Em contrapartida, no ambiente dos videogames, o modding assumiu o sentido original da expressão hacking, se referindo à ação do usuário realizar modificações personalizadas ao manipular a estrutura de funcionamento do jogo, alterando elementos como texturas de cenários e de roupas de personagens, inserindo novos objetos gráficos, criando novos mapas exploráveis ou um jogo com mecânicas distintas do original. Isso pode ser feito através de alterações diretas no código fonte do jogo, ou por ferramentas criadas pelos próprios desenvolvedores, a fim de incentivar a criatividade de seu público de forma mais ampla (SOTAMMA, 2003). O modding seria uma forma de hacking sobre o jogo, mas com uma finalidade de entretenimento, em que o usuário assume o papel de game designer e interfere na visualidade da obra e em seu funcionamento. Uma prática que, como aponta Champion (2012, p. 12, tradução nossa), atualmente tem como principal foco o ato de "alterar o gameplay e a experiência do jogo em si" ${ }^{\prime 13}$, invés de ser algo incremental ao visual do jogo original.

Scacchi (2010) categoriza outras práticas de modding além das citadas anteriormente, como a interferência nas estéticas e nas funcionalidades de hardwares de computadores e consoles, bem como o Machinima - produções audiovisuais geradas a partir de jogos ${ }^{14}$, e Art

\footnotetext{
${ }^{13}$ No original: $[\ldots]$ change to the gameplay and the experience of the game itself.

${ }^{14}$ Como a série cômica Red vs Blue, derivada dos jogos da franquia Halo, lançada no YouTube em 2002 pela produtora Rooster Teeth e atualmente na sua quinta temporada. Disponível em:

https://www.youtube.com/channel/UCIIOhP2Ycmhh5j81S4cexBQ. Acesso em: 14 jan. 2018.
} 
Mods - trabalhos artísticos que surgem a partir da manipulação da jogabilidade pelo hacking das regras do jogo e pela intervenção na sua visualidade original ${ }^{15}$. $\mathrm{O}$ autor encara o modding como uma forma de personalização tecnológica que abre possibilidades de inovação sóciotécnicas, baseando-se na apropriação do design dos produtores originais e suas potencialidades como linguagem e agentes nas relações sociais e comerciais.

O fator inovador na modificação realizada em TwicthPlaysPokemon encontra-se no fato de que o hacking ocorreu através da Twitch.TV, que não apenas transmite partidas do jogo como também passar a ser uma plataforma de jogo, utilizando-se do espaço de bate-papo como input das ações desejadas (controle). Ao integrar o jogo e o serviço de vídeo sob demanda, a mediação textual passa a ter um papel de controle da experiência. Inicialmente, a personagem do videogame obedecia a todos os comandos de texto digitados pelos usuários, tais como up, down, select, etc. (Fig. 3). Após a primeira semana do canal, foi instaurado o modo de jogo 'democracia', em que os comandos executados eram votados a partir de uma apuração automática realizada de cinco em cinco segundos. Também havia a possibilidade de votar, em intervalos entre 10 e 15 minutos, por instaurar o modo 'anárquico' - no qual a escolha de comandos se dá de modo aleatório.

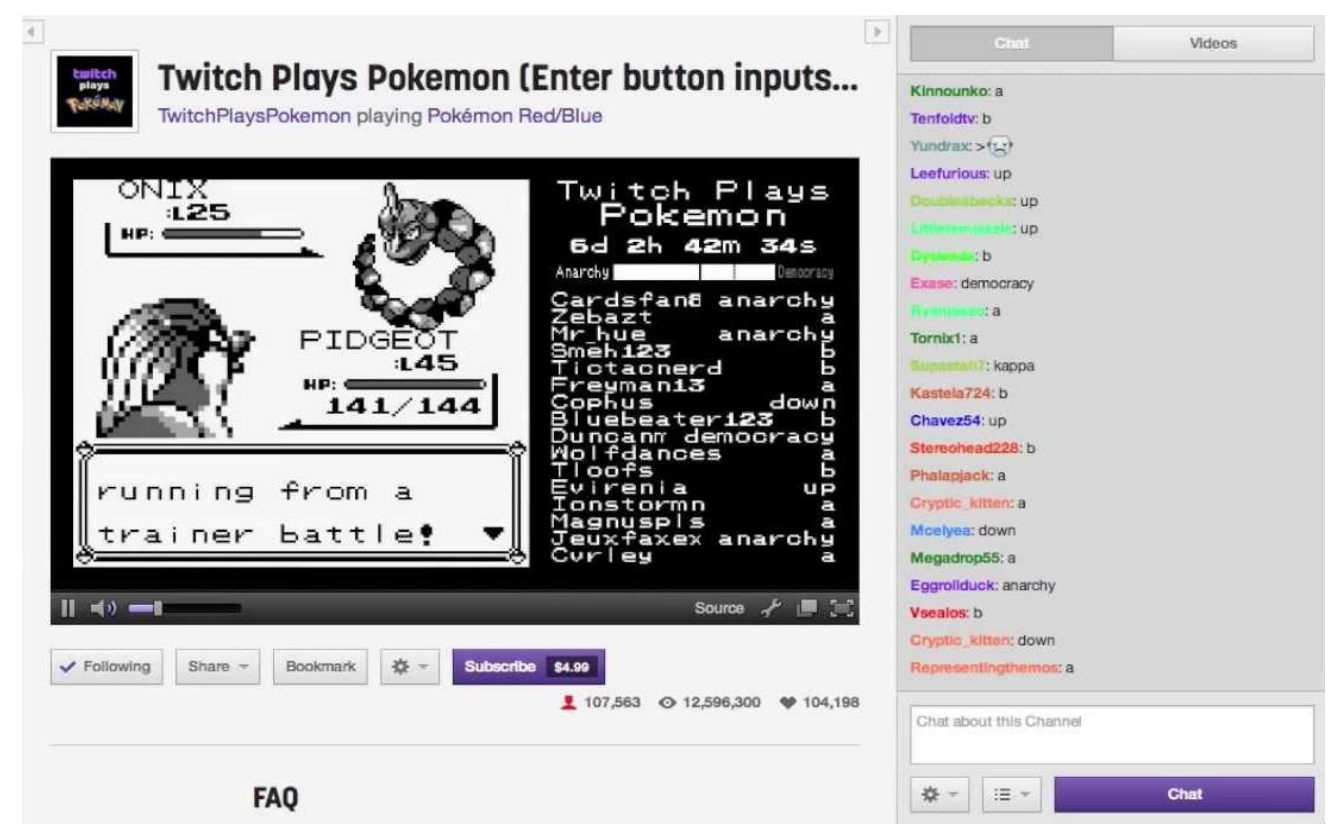

Figura 3 - Detalhe da interface de transmissão do primeiro jogo transmitido pelo canal em 2014, com Pokémon Red (1996), tendo à direita os comandos de textos digitados na sala de bate-papo.

Fonte: https://i.ytimg.com/vi/0QSFavoEU38/maxresdefault.jpg. Acesso em: 10 dez. 2018

\footnotetext{
15 Scacchi (2010) destaca exposições de arte de modificações de jogos como a "Shift-Cntl", realizada em 2000 pela Beall Center for Art + Technology, na Califórnia.
} 
A fim de comportar os debates promovidos pelos usuários, foi criado o fórum ${ }^{16}$ do canal da plataforma Reddit ${ }^{17}$. Isso contribuiu para formar uma identidade do agrupamento de usuários, bem como para "recrutar" desenvolveres para contribuir com o projeto. Como aponta Ramirez (et al., 2014), no início do canal foram formados dois grupos polarizados de jogadores em torno da experiência. O primeiro grupo, composto por um número menor de usuários, acreditava que caos seria essencial para experiência, pois o acaso e o improviso seriam elementos inerentes à participação no bate-papo da Twitch.TV, e isso deveria refletir no jogo em si. O segundo grupo, queria impor uma ordem clara à experiência do jogo, para que ele fosse terminado apropriadamente e a transmissão não fosse considerada uma perda de tempo e de esforço para os usuários. O conflito entre tais dinâmicas fica claro pelo comentário do usuário WhatHappedToEvaXep no fórum online ${ }^{18}$ :

Quando "Democracia" foi introduzida, pensei que era a solução perfeita para nossos problemas. Sem mais ter que ficar olhando para a tela de pause [...], sem mais acidentes no PC, sem mais compras ruins no Pokemart...

Quando a Democracia era confrontada com "tumultos" na sala de bate-papo e "protestos" sob a forma do spam start $9^{19}$, eu não conseguia entender por que tantas pessoas foram contra isso. A democracia não seria mais inteligente? Não seria mais eficiente? Não seria mais eficaz?

Sim, seria mais inteligente. Sim, seria mais eficiente. Sim, seria mais eficaz. E é exatamente isso que está errado.

Eu percebi que a razão pela qual muitas pessoas estão curtindo Twitch Plays Pokemon é porque é imprevisível e caótico. É divertido observar o resultado de dezenas de milhares de pessoas pressionando botões. É divertido ver o que os eventos loucos acontecem a seguir.

Se o jogo estava sendo jogado com apenas o botão "correto" pressionado, então todos os memes que desfrutamos nunca teriam surgido. ${ }^{20}$ (tradução nossa).

\footnotetext{
${ }^{16}$ Conferir: https://www.reddit.com/r/twitchplayspokemon/. Acesso em: 7 set. 2018.

${ }^{17}$ Rede social lançada em 2005 que funciona como um agregador de fóruns online, divididos em diversas categorias de interesse criadas pelos usuários.

${ }^{18}$ Disponível em:

https://www.reddit.com/r/twitchplayspokemon/comments/1yir32/i_was_a_diehard_supporter_of_democracy_but _now_i/. Acesso em: 15 jan. 2018. Tradução livre.

${ }^{19}$ Comando para pausar o jogo, tornando o tempo do jogo no modo democracia menor.

${ }^{20}$ No original: When "Democracy" was introduced, I thought that it was the perfect solution to our woes. No more staring at the pause screen, no more falling off ledges, no more accidents at the PC, no more bad purchases at the Pokemart...

When Democracy was met with "riots" in the chatroom and "protests" in the form of start9 spam, I couldn't understand why so many people were opposed to it. Wouldn't democracy be smarter? Wouldn't it be more efficient? Wouldn't it be more effective?

Yes, it would be smarter. Yes, it would be more efficient. Yes, it would be more effective. And that's exactly what's wrong with it.

I've realized that the reason so many people are enjoying Twitch Plays Pokemon is because it's unpredictable and chaotic. It's entertaining to watch the outcome of tens of thousands of people pressing buttons. It's entertaining to see what crazy events happens next.
} 
O fórum demarca fortemente esse espaço de disputa entre as visões do público do canal, além de prover um ambiente para discutir sobre as funcionalidades e implicações do projeto, ou mesmo para divulgar ações relacionados ao universo de pokémon. O poder assumido pelo público possui é tão evidente que, em novembro de 2017 , o criador do canal (um usuário anônimo conhecido apenas como "Streamer Original") decidiu abandonar sua posição de liderança ${ }^{21}$, delegando-a a outro usuário. Isso ocorreu após atritos com jogadores diante de suas ações autoritárias sobre o projeto (banindo alguns por discordarem de suas decisões), chegando ao ponto de divulgar informações pessoais de um usuário devido a um desentendimento (JACKSON, 2017). Isso demonstra mais uma forma de relação da cultura participativa com TwicthPlaysPokemon, a partir dos fundamentos da ética hacker de se contrapor à autoridade e apoiar a descentralização do poder - em que o público tem influência e diálogo direto com os produtores de conteúdos.

Além de representar essa dimensão simbólica, o hacking articulou e integrou o design de duas plataformas de mídias distintas. Foi conjugada a visualidade de uma plataforma de vídeo como o Twicth com os desafios do universo ficcional dos jogos da franquia Pokémon. Trata-se de uma proposta de prática transmídia (DENA, 2009), em que processos de adaptações são capazes de trazer novos significados e experiências ao público em relação às versões originais. Afinal, segundo a autora, o mais estratégico na convergência é reconhecer que cada meio possui sua própria linguagem e designs distintos, com suas possibilidades de funcionalidades complementares. Cabe aos produtores e ao público reconhecerem as características de cada mídia e articular possíveis integrações pelos conteúdos, mesmo que via hacking, como visto. Mas, mais que uma adaptação ou uma prática transmídia, TwicthPlaysPokemon criou o que podemos considerar como uma experiência colaborativa massiva, em que decisões tomadas foram negociadas pelos próprios participantes, e que, mesmo havendo diferentes interesses em jogo, seguiu-se o princípio da manutenção de um ambiente lúdico. Diferentemente de outros jogos massivos online, em que usuários interpretam apenas um avatar por vez, em TwicthPlaysPokemon todos os usuários encaram o mesmo avatar, de forma simultânea, intensificando uma dimensão participativa e

If the game was being played with only the "correct" button presses, then all of the memes we've been enjoying would never have come into existence.

21 O comunicado oficial pode ser lido pelo link:

https://www.reddit.com/r/twitchplayspokemon/comments/7d0w62/official_transfer_of_leadership_of_the_t witch/. Acesso em: 14 jan. 2018. 
proporcionando novos significados para o ato jogar - como pôde ser visto pelo debate entre uma jogabilidade democrática ou anárquica.

\section{CONSIDERAÇÕES FINAIS}

O modding é uma prática que acompanha os jogos, e um exemplo de cultura participativa, mas, em TwicthPlaysPokemon, esse processo se intensifica e rompe com o modelo tradicionalmente observado. Ramirez (et al., 2014), compreende o fenômeno TwicthPlaysPokemon como uma forma de experimento social (não apenas tecnológico), caracterizado por uma variedade de vozes e interpretações que circulam por agrupamentos efêmeros que se formam a cada partida, ou mesmo pela comunidade que se formou ao longo do tempo. Também, podemos considerar esse canal como um caso representativo das práticas da cultura participativa e da convergência tecnológica de mídias - neste último caso, materializadas pelo hibridismo do videogame com o vídeo sob demanda - em uma apropriação por parte do público de obras da franquia Pokémon, desde sua concepção até sua recepção pela plataforma Twitch.TV.

Porém, é preciso considerar que as relações entre usuários, mesmo em um agrupamento tão específico, são marcadas por conflitos e (re)negociações de sentido. No viés da convergência, o hacking na plataforma Twicth.TV viabilizou um modo distinto de experiência tanto para o público de jogadores quanto para os usuários do serviço de vídeo (espectadores que assistem "em silêncio" a uma partida coletiva em andamento). A manipulação de funcionalidades trouxe novos significados, possibilidades e regimes de interações aos jogadores, mesmo àqueles mais habituados a franquia Pokémon.

A experiência pode servir de base para explorar outras funcionalidades potenciais entre mídias distintas, tornando evidente o cruzamento de vozes que está presente na lógica da cultura participativa - que deve, idealmente, conduzir para o diálogo a partir da diferença. No campo de estudos de video games, evidencia-se pesquisas que possam focar, principalmente, nas ferramentas que constituem a participação contemporânea e como diferentes subjetividades podem surgir do modo como o design da plataforma participativa é planejado, ou retrabalhado através da ação dos usuários. São subjetividades que, quando interagem de forma tão múltipla, desenvolvem o potencial de transformação de qualquer forma de experiência narrativa e lúdica previamente traçada pelo criador original, sendo incorporadas 
por uma espécie de corpo político colaborativo que tenta mediar os desdobramentos de um mundo ficcional dotado de vários caminhos possíveis.

\section{REFERÊNCIAS}

CERETTA, F. M. A Discussão Política por Meio de Memes: o caso de Eduardo Cunha e a apropriação cômica das redes. In: IX Simpósio Nacional ABCiber, 2016, PUCSP. Anais... São Paulo: PUCSP, 2016

CHAMPION, E. et al. Game mods: theory and criticism. Morrisville: lulu.com, 2013.

DENA, C. Transmedia Practice: Theorising the Practice of Expressing a Fictional World across Distinct Media and Environments. Sydney: University of Sydney, 2009. (Tese de Doutorado).

GOOD, O. Fan-made Pokemon Uranium is shelved by its creators after Nintendo notices. Polygon, 10 dez. 2017. Disponível em: https://www.polygon.com/2016/8/14/12472616/pokemon-uranium-takendown-nintendo. Acesso em: 08 dez. 2017.

GREGOLIN, M. Machinima e a era digital: produto da contemporaniedade. São Carlos: Claraluz, 2010.

GUSMÃO, G. Partida coletiva de Pokémon reúne 100 mil jogadores, sobrecarrega Twitch e gera subcultura na web. EXAME, 20 fev. 2014. Disponível em:

https://exame.abril.com.br/tecnologia/partida-coletiva-de-pokemon-reune-ate-100-mil-jogadoressobrecarrega-twitch-e-gera-subcultura-na-web/. Acesso em: 06 out. 2017.

JACKSON, G. Twitch Plays Pokémon's Creator Says He Is Stepping Down Following Alleged Doxing. Kotaku, 15 nov. 2017. Disponível em: https://kotaku.com/twitch-plays-pokemon-s-creatorsays-he-is-stepping-down-1820486151. Aceso em: 12 jan. 2018.

JENKINS, H. Interactive Audiences?: The 'Collective Intelligence' of Media Fans. Henry Jenkins Blog, 2003. Disponível em: http://web.mit.edu/21fms/People/henry3/collective\%20intelligence.html. Acesso em: 07 dez. 2017.

, H. Cultura da Convergência. 1 ed. São Paulo: Aleph, 2009.

, H. Transmedia 202: Further Reflections. Confessions of an Aca-fan, 31 jul. 2011.

Disponível em: http://henryjenkins.org/blog/2011/08/defining_transmedia_further_re.html. Acesso em: 07 dez. 2017.

, H.; FORD, S.; GREEN, J. Cultura da Conexão: criando valor e significado por meio da mídia propagável. São Paulo: Aleph, 2014.

SCACCHI, W. Computer game mods, modders, modding, and the mod scene. First Monday, n. 5, v. 5, mai. 2010. Disponível em: 〈http://uncommonculture.org/ojs/index.php/fm/article/view/2965〉. Acesso em: 14 jan. 2018.

SOTAMMA, O. Computer Game Modding, Intermediality, and Participatory Culture. New Media, v. 5, n. 1. Disponível em:

http://www.yorku.ca/caitlin/futurecinemas/coursepack2009/Sotamaa_modding.pdf. Acesso em: 08 dez. 2017. 
HAMILTON, W., GARRETSON, O., KERNE, A. 2014. Streaming on twitch: fostering participatory communities of play within live mixed media. In: Proceedings of the SIGCHI Conference on Human Factors in Computing Systems (CHI '14). ACM, Anais..., New York, NY, USA, 1315-1324. DOI: https://doi.org/10.1145/2556288.2557048.

MACHADO, M. B. Por dentro dos Anonymous Brasil: poder e resistência na sociedade de controle. Santo André: UFABC, 2010. 120 f. Dissertação (Mestrado em Ciências Humanas e Sociais.

NEDELCHEVA, I. Analysis of Transmedia Storytelling in Pokémon GO. International Science Index, Humanities and Social Sciences, v. 10, n. 11, 2016. Disponível em:

http://waset.org/publications/10006132/analysis-of-transmedia-storytelling-in-pok\%C3\%A9mon-go. Acesso em: 08 dez. 2017

\section{Original recebido em: 10 de dezembro de 2018}

Aceito para publicação em: 14 de julho de 2019

Dario Mesquita

Doutorando em Design pela Universidade Anhembi Morumbi, mestre em Imagem e Som pela UFSCar. Professor Adjunto do Departamento de Artes e Comunicação - DAC/UFSCar. Pesquisador do grupo GEMInIS.

Sérgio Nesteriuk

Professor e coordenador do PPG Design da Universidade Anhembi Morumbi. Líder do DEED

- Grupo de Pesquisa em Design, Entretenimento e Educação. E-mail: nesteriuk@hotmail.com

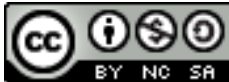

Esta obra está licenciado com uma Licença

Creative Commons Atribuição - Não Comercial - Compartilha Igual 4.0 Internacional 